\title{
Density Functional Theory Analysis of Gas Adsorption on Monolayer and Few Layer Transition Metal Dichalcogenides: Implications for Sensing
}

\author{
Vasudeo Babar`, Hakkim Vovushađ, and Udo Schwingenschlögl* \\ King Abdullah University of Science and Technology (KAUST), \\ Physical Science and Engineering Division (PSE), Thuwal 23955-6900, Saudi Arabia
}

\begin{abstract}
First principles calculations are performed to compare the adsorption of $\mathrm{CO}, \mathrm{NH}_{3}, \mathrm{NO}$, and $\mathrm{NO}_{2}$ molecules on monolayer, bilayer, and heterobilayer $\mathrm{MoS}_{2}$ and $\mathrm{WS}_{2}$, using van der Waals corrected density functional theory. Only minor differences are demonstrated for the adsorption behaviors of the monolayer and bilayer systems despite fundamental differences in the electronic structure (direct versus indirect band gap). We also show that $\mathrm{NO}_{2}$ binds stronger to the sensor materials than the other gas molecules, resulting in enhanced charge transfer. Adsorption of paramagnetic $\mathrm{NO}$ and $\mathrm{NO}_{2}$ has significant impact on the electronic states, in contrast to adsorption of nonmagnetic $\mathrm{CO}$ and $\mathrm{NH}_{3}$.
\end{abstract}

Keywords: sensor, gas, transition metal dichalcogenide, adsorption, first principles

\footnotetext{
I Equal contribution

*udo.schwingenschlogl@kaust.edu.sa
} 


\section{INTRODUCTION}

Metal oxides are conventional gas sensing materials with high sensitivity at low cost [15]. However, they often require high operating temperatures, about $200-500{ }^{\circ} \mathrm{C}$, causing issues of power consumption and safety $[6,7]$. Development of gas sensing materials with high sensitivity that can operate at room temperature therefore is of great importance, for example in areas such as human health, agriculture, and industry [8-11]. In this context, two-dimensional materials are attractive candidates, particularly transition metal dichalcogenides $\left(\mathrm{MoS}_{2}, \mathrm{WS}_{2}\right.$, etc.), as they provide a wide range of band gap sizes [12-14]. Gas sensing by two-dimensional materials typically exploits charge transfer processes that modify the resistance after exposure to gas molecules [15]. For example, a field effect transistor based on monolayer $\mathrm{MoS}_{2}$ can provide excellent detection limits of 1 ppm for $\mathrm{NH}_{3}$ and 20 ppb for $\mathrm{NO}_{2}$ [16]. monolayer $\mathrm{MoSe}_{2}$ has been used to sense $\mathrm{NH}_{3}$ with a detection limit of 50 ppm [17]. A field effect transistor based on graphene for sensing $\mathrm{CO}, \mathrm{NH}_{3}, \mathrm{NO}_{2}$, and $\mathrm{H}_{2} \mathrm{O}$ at room temperature has been discussed in Ref. [18], sensing of $\mathrm{NH}_{3}$ and $\mathrm{NO}_{2}$ by monolayer $\mathrm{MoS}_{2}$ has been studied by in-situ photoluminescence spectroscopy in Ref. [19], and it has been demonstrated that monolayer $\mathrm{MoS}_{2}$ supported on $\mathrm{SiO}_{2} / \mathrm{Si}$ substrate provides selective response to electron donor analytes [20].

While the preparation of monolayer transition metal dichalcogenides is expensive, few layer films provide also high surface-to-volume ratios and can be obtained by scalable methods [21]. However, in contrast to the direct band gaps of monolayer transition metal dichalcogenides, bilayers and few layer films reproduce the main features of the bulk band structures (in particular the indirect band gap nature) $[22,23]$. Because of this fundamental difference in the electronic structure, it cannot be predicted whether the excellent gas sensing properties of monolayer transition metal dichalcogenides are inherited by few layer films. Density functional theory previously has been used to investigate the adsorption of gas molecules on monolayer $\mathrm{MoS}_{2}$ [24], monolayer $\mathrm{WS}_{2}$ [25], and monolayer $\mathrm{MoS}_{2} / \mathrm{WS}_{2}$ in-plane heterojunctions [26]. In the present study, we perform a systematic first principles investigation to establish the adsorption behavior of prototypical gas molecules $\left(\mathrm{CO}, \mathrm{NH}_{3}, \mathrm{NO}\right.$, and $\mathrm{NO}_{2}$ ) on $\mathrm{MoS}_{2}$ and $\mathrm{WS}_{2}$, comparing the monolayer geometry (direct band gaps) to few layer films (which we represent by bilayers, as these already realize indirect band gaps). We also discuss results for $\mathrm{MoS}_{2} / \mathrm{WS}_{2}$ heterobilayers. Adsorption energies, Bader charges, and 
electronic band structures are used to describe the molecule-sensor interaction.

\section{COMPUTATIONAL METHODS}

We use spin-polarized density functional theory as implemented in the Vienna Ab-initio Simulation Package [27] with the generalized gradient approximation for the exchangecorrelation potential (Perdew-Burke-Ernzerhof parametrization). The plane wave energy cutoff is set to $500 \mathrm{eV}$, the van der Waals interaction is described by the DFT-D2 method [28], and the Brillouin zone is sampled on $3 \times 3 \times 1 \mathrm{k}$-meshes. Moreover, the adsorption energy of gas molecules,

$$
E_{a}=E[\text { sensor }]+E[\text { molecule }]-E[\text { sensor }+ \text { molecule }],
$$

is calculated from the total energies before and after adsorption. Positive values imply that adsorption is energetically favorable. We use hexagonal $4 \times 4 \times 1$ supercells (lattice constant $12.73 \AA$ ) of monolayer $\mathrm{MoS}_{2}$ and $\mathrm{WS}_{2}$, consisting of 48 atoms, and periodic boundary conditions with a $15 \AA$ vacuum slab added in the $c$-direction. bilayers and heterobilayers are constructed for different stacking configurations (96 atoms per supercell). It turns out that location of the $\mathrm{S}$ atoms of one layer on top of the Mo/W atoms of the other layer minimizes the total energy, in agreement with previous reports [29-31]. This stacking configuration therefore is used throughout our study. Structural optimizations are conducted by the conjugate gradient method until the changes in the total energy and atomic forces decline below $10^{-6} \mathrm{eV}$ and $5 \times 10^{-3} \mathrm{eV} \AA^{-1}$, respectively. The charge transfers between the gas molecules and sensor materials are calculated by Bader charge analysis [32].

\section{RESULTS AND DISCUSSION}

The favorable adsorption sites of the gas molecules are taken from previous studies on monolayer $\mathrm{MoS}_{2}$ [24] and monolayer $\mathrm{WS}_{2}$ [25]. The relaxed structures obtained after adding one $\mathrm{CO}, \mathrm{NH}_{3}, \mathrm{NO}$, or $\mathrm{NO}_{2}$ molecule to monolayer $\mathrm{MoS}_{2}$ are shown in Figure 1(left). Qualitatively the same structures are found for adsorption on monolayer $\mathrm{WS}_{2}$. We obtain for the $\mathrm{CO}, \mathrm{NH}_{3}, \mathrm{NO}, \mathrm{NO}_{2}$ molecules distances of $3.48,3.28,3.15,3.25 \AA$ from monolayer $\mathrm{MoS}_{2}$ and distances of $3.43,3.28,3.14,3.21 \AA$ from monolayer $\mathrm{WS}_{2}$, respectively. The computed 


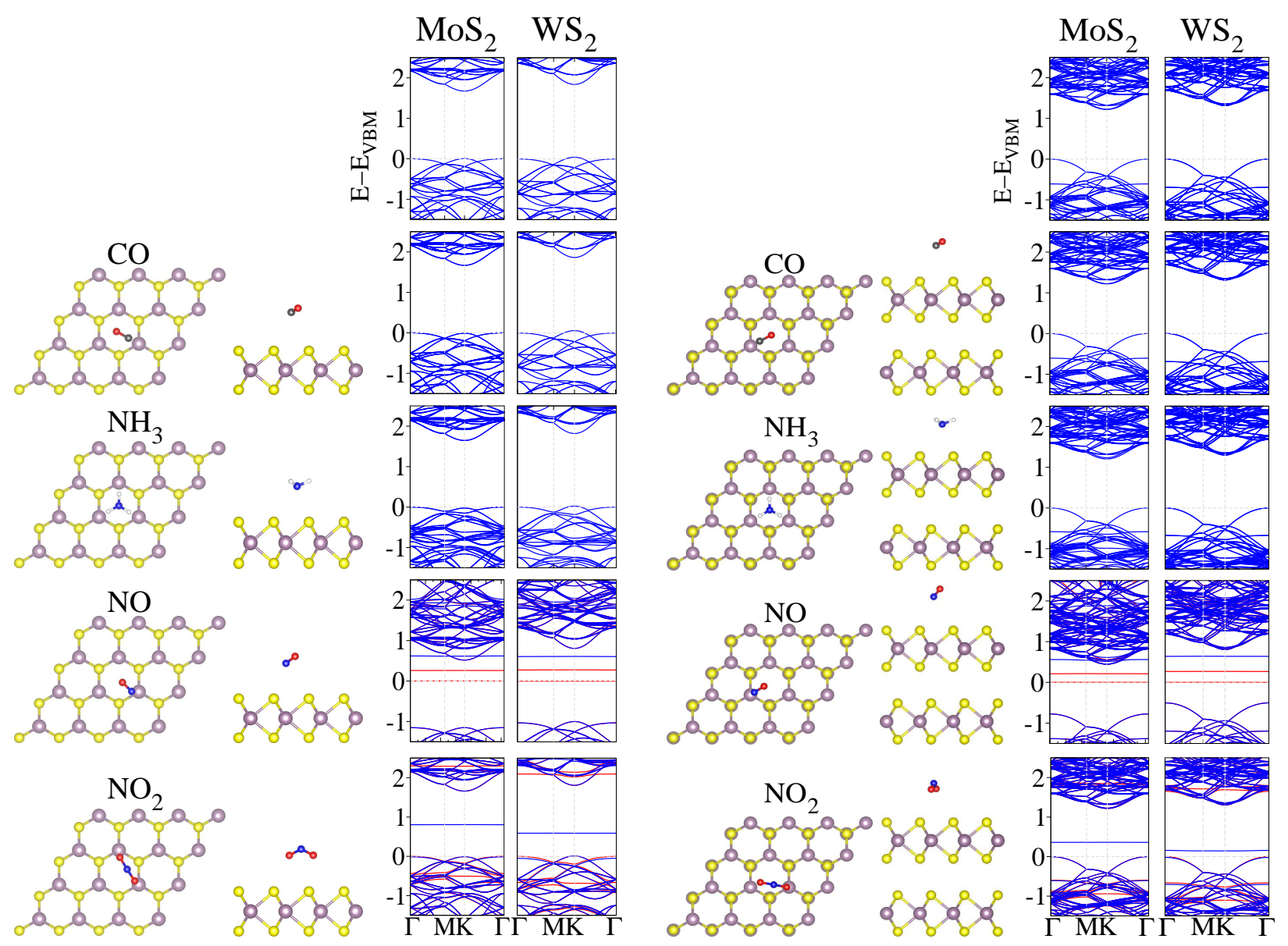

FIG. 1. Top and side views of relaxed monolayer (left) and bilayer (right) $\mathrm{MoS}_{2}$ with different molecules adsorbed (white: H, black: C, blue: N, red: O, yellow: S, pink: Mo/W). Qualitatively the same structures are obtained for $\mathrm{WS}_{2}$. Band structures are given for adsorption on both $\mathrm{MoS}_{2}$ and $\mathrm{WS}_{2}$. Corresponding results for the pristine materials are shown on top.

adsorption energies of the gas molecules on monolayer $\mathrm{MoS}_{2}$ and $\mathrm{WS}_{2}$ are summarized in Figure 2. It turns out that they are systematically higher on monolayer $\mathrm{WS}_{2}$ than on monolayer $\mathrm{MoS}_{2}$, in agreement with previous results [33]. We find that $\mathrm{CO}, \mathrm{NH}_{3}, \mathrm{NO}$, and $\mathrm{NO}_{2}$ donate $0.01(0.04), 0.06(0.05), 0.00(-0.03)$, and $-0.11(-0.07)$ electrons to monolayer $\mathrm{MoS}_{2}$ $\left(\mathrm{WS}_{2}\right)$, respectively. According to Figure 2, all gas molecules are physisorbed on monolayer $\mathrm{MoS}_{2}$ and $\mathrm{WS}_{2}$, where the slightly enhanced adsorption energy of $\mathrm{NO}_{2}$ corresponds to the observation of higher charge transfer to the sensor material. The $\mathrm{C}$ atom of $\mathrm{CO}, \mathrm{N}$ atoms of $\mathrm{NH}_{3}$ and $\mathrm{NO}$, and $\mathrm{O}$ atom of $\mathrm{NO}_{2}$ point towards the sensor material. We find that the band gaps of monolayer $\mathrm{MoS}_{2}$ and $\mathrm{WS}_{2}$ amount to 1.66 and $1.79 \mathrm{eV}$, respectively, matching 


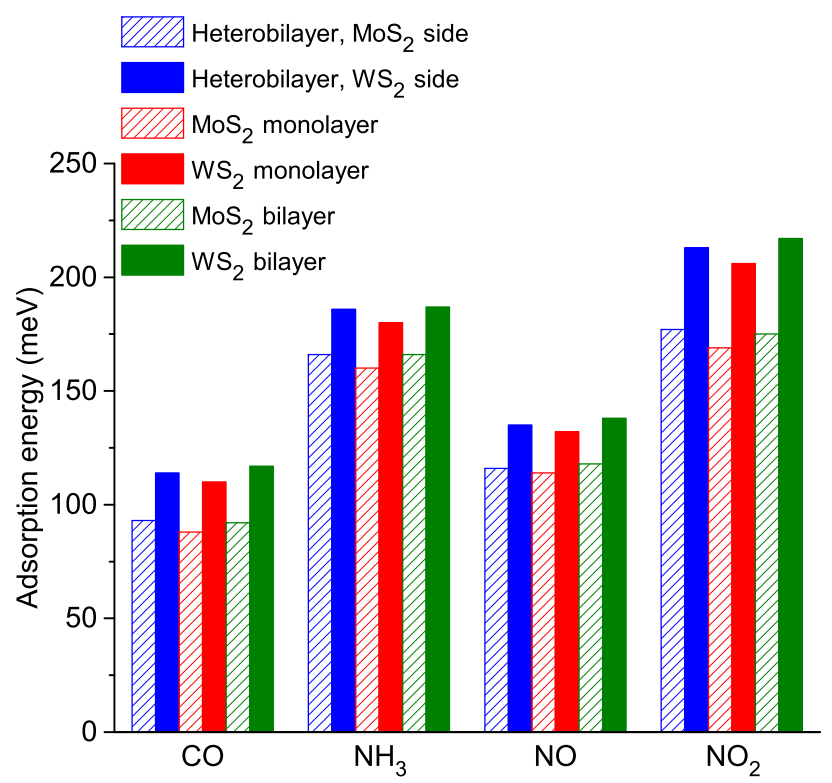

FIG. 2. Comparison of the adsorption energies obtained for adsorption of $\mathrm{CO}, \mathrm{NH}_{3}, \mathrm{NO}$, and $\mathrm{NO}_{2}$ molecules on monolayer, bilayer, and heterobilayer $\mathrm{MoS}_{2}$ and $\mathrm{WS}_{2}$.

well with earlier reports [24, 25]. According to the band structures given in Figure 1(left) with respect to the valence band maximun (VBM), the valence and conduction bands are hardly affected by the adsorption of non-magnetic $\mathrm{CO}$ and $\mathrm{NH}_{3}$, while the adsorption of paramagnetic $\mathrm{NO}$ and $\mathrm{NO}_{2}$ on monolayer $\mathrm{MoS}_{2}\left(\mathrm{WS}_{2}\right)$ introduces two (three) and one (one) flat impurity bands inside the band gap, respectively.

Figure 1(right) shows the relaxed structures obtained after adsorption of the gas molecules on bilayer $\mathrm{MoS}_{2}$. Qualitatively the same structures are found for adsorption on bilayer $\mathrm{WS}_{2}$. In addition, the same molecular orientations are favorable as on the monolayers. It turns out that the same adsorption sites are energetically favorable for the bilayers as for the monolayers. In addition, the distances of the $\mathrm{CO}, \mathrm{NH}_{3}, \mathrm{NO}, \mathrm{NO}_{2}$ molecules from bilayer $\mathrm{MoS}_{2}(3.46,3.29,3.16,3.26 \AA)$ and bilayer $\mathrm{WS}_{2}(3.42,3.27,3.14,3.23)$ are almost identical to those reported above for the monolayers. Corresponding adsorption energies are given in Figure 2. Bilayer $\mathrm{WS}_{2}$ provides systematically stronger interaction than bilayer $\mathrm{MoS}_{2}$. In addition, for each molecule the interaction is stronger on the bilayers than on the monolayers. For bilayer $\mathrm{MoS}_{2}\left(\mathrm{WS}_{2}\right)$ we find that $\mathrm{CO}, \mathrm{NH}_{3}, \mathrm{NO}$, and $\mathrm{NO}_{2}$ donate -0.03 (0.03), 0.00 (0.02), $-0.02(-0.01)$, and $-0.08(-0.07)$ electrons, respectively. The band gaps of bilayer $\mathrm{MoS}_{2}$ and $\mathrm{WS}_{2}$ amount to 1.22 (indirect) and $1.31 \mathrm{eV}$ (indirect), respectively, in agreement 


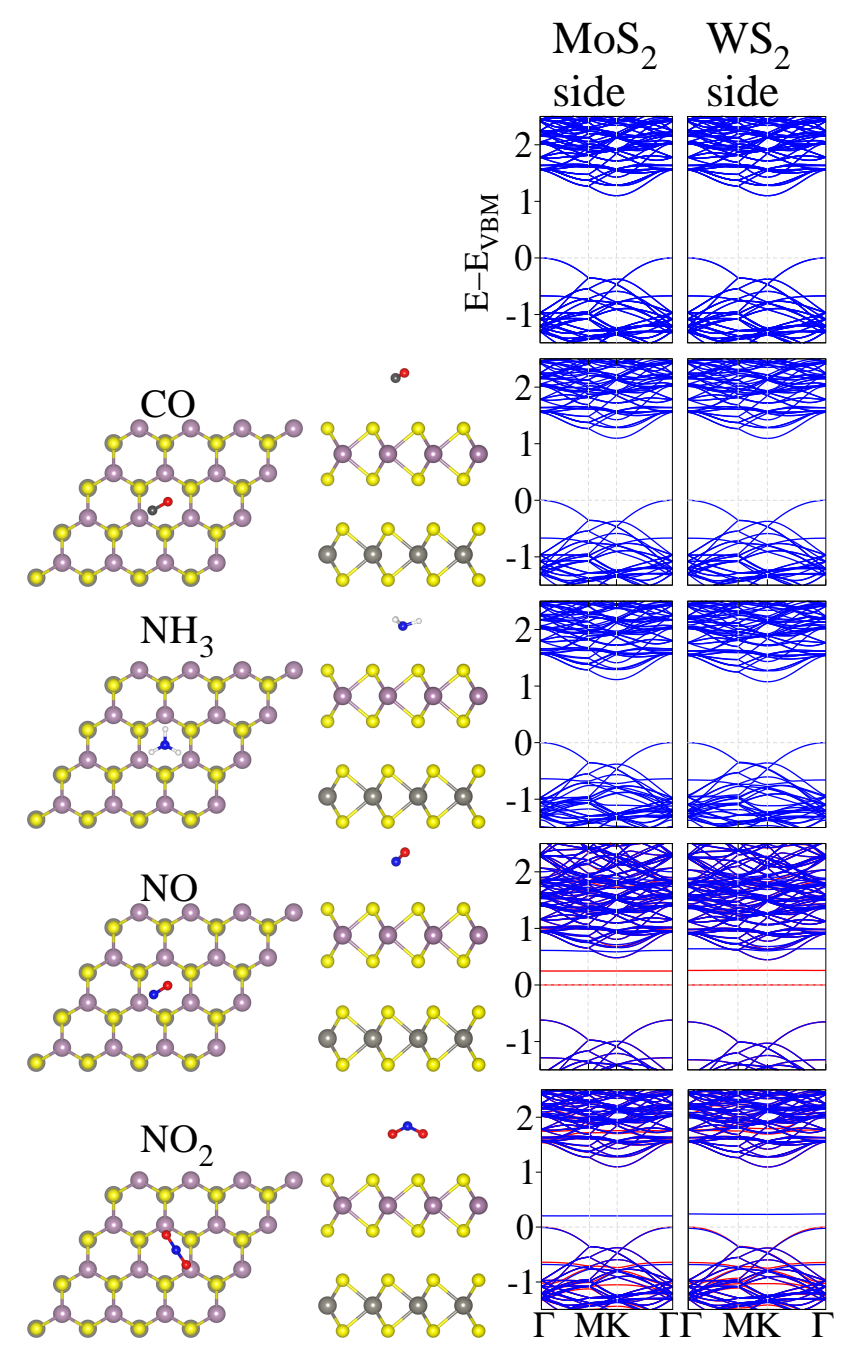

FIG. 3. Top and side views of relaxed $\mathrm{MoS}_{2} / \mathrm{WS}_{2}$ heterobilayers with different molecules adsorbed on the $\mathrm{MoS}_{2}$ side (white: H, black: C, blue: N, red: O, yellow: S, pink/grey: Mo/W). Qualitatively the same structures are obtained for adsorption on the $\mathrm{WS}_{2}$ side of the heterobilayer. Band structures are given for adsorption on both the $\mathrm{MoS}_{2}$ and $\mathrm{WS}_{2}$ sides of the heterobilayer. Corresponding results for the pristine heterobilayer are shown on top.

with Ref. [31]. According to Figure 1(right), the adsorption of non-magnetic $\mathrm{CO}$ and $\mathrm{NH}_{3}$ has no relevant influence on the conduction and valence bands, while the adsorption of paramagnetic $\mathrm{NO}$ and $\mathrm{NO}_{2}$ on bilayer $\mathrm{MoS}_{2}\left(\mathrm{WS}_{2}\right)$ introduces two (three) and one (one) flat impurity bands inside the band gap, respectively.

For comparison with the monolayers and bilayers, we also study a $\mathrm{MoS}_{2} / \mathrm{WS}_{2}$ heterobilayer. The interlayer distance in the heterobilayer turns out to be $6.16 \AA$, in agreement with 
TABLE I. Adsorption energies (in $\mathrm{meV}$ ) of $\mathrm{CO}, \mathrm{NH}_{3}, \mathrm{NO}$, and $\mathrm{NO}_{2}$ on the sensor materials under consideration, compared to literature results for selected two-dimensional materials.

\begin{tabular}{|c|c|c|c|c|c|c|}
\hline & $\mathrm{CO}$ & $\mathrm{NH}_{3}$ & $\mathrm{NO}$ & $\mathrm{NO}_{2}$ & Method & Reference \\
\hline monolayer $\mathrm{MoS}_{2}$ & 88 & 160 & 114 & 169 & DFT-D2 & This work \\
\hline monolayer $\mathrm{WS}_{2}$ & 110 & 180 & 132 & 206 & DFT-D2 & This work \\
\hline bilayer $\mathrm{MoS}_{2}$ & 92 & 166 & 118 & 175 & DFT-D2 & This work \\
\hline bilayer $\mathrm{WS}_{2}$ & 117 & 187 & 138 & 217 & DFT-D2 & This work \\
\hline $\mathrm{MoS}_{2} / \mathrm{WS}_{2}$ heterobilayer, $\mathrm{MoS}_{2}$ side & 93 & 166 & 116 & 177 & DFT-D2 & This work \\
\hline $\mathrm{MoS}_{2} / \mathrm{WS}_{2}$ heterobilayer, $\mathrm{WS}_{2}$ side & 114 & 186 & 135 & 213 & DFT-D2 & This work \\
\hline monolayer $\mathrm{MoS}_{2}$ & 128 & 250 & 211 & 276 & LDA & {$[24]$} \\
\hline monolayer $\mathrm{MoS}_{2}$ & 13 & 44 & 14 & 43 & GGA & {$[24]$} \\
\hline monolayer $\mathrm{WS}_{2}$ & 127 & 216 & 215 & 412 & GGA & {$[25]$} \\
\hline Graphene & 14 & 31 & 29 & 67 & GGA & {$[35]$} \\
\hline h-BN & 97 & 100 & 117 & 125 & LDA & {$[36]$} \\
\hline $\mathrm{MoS}_{2} / \mathrm{WS}_{2}$ in-plane heterojunction & 120 & 186 & 216 & 550 & LDA & {$[26]$} \\
\hline $\mathrm{MoS}_{2} / \mathrm{WS}_{2}$ in-plane heterojunction & 1 & 28 & 21 & 28 & GGA & {$[26]$} \\
\hline $\mathrm{MoS}_{2} / \mathrm{WS}_{2}$ in-plane heterojunction & 90 & 158 & 122 & 161 & DFT-D2 & {$[26]$} \\
\hline
\end{tabular}

Ref. [34]. Figure 3 shows the structures obtained for adsorption of the molecules on the $\mathrm{MoS}_{2}$ side of the heterobilayer. Adsorption on the $\mathrm{WS}_{2}$ side of the heterobilayer results in qualitatively the same structures. Again the $\mathrm{C}$ atom of $\mathrm{CO}, \mathrm{N}$ atoms of $\mathrm{NH}_{3}$ and $\mathrm{NO}$, and $\mathrm{O}$ atom of $\mathrm{NO}_{2}$ point towards the sensor material. The distances between the closest atoms of the gas molecules and sensor material turn out to be $3.48,3.29,3.24$, and $3.25 \AA$ (3.41, $3.27,3.14$, and $3.22 \AA$ ) for $\mathrm{CO}, \mathrm{NH}_{3}, \mathrm{NO}$, and $\mathrm{NO}_{2}$ adsorbed on the $\mathrm{MoS}_{2}\left(\mathrm{WS}_{2}\right)$ side of the heterobilayer, respectively. The corresponding adsorption energies, see Figure 2, are higher for adsorption on the $\mathrm{WS}_{2}$ than on the $\mathrm{MoS}_{2}$ side. We find that $\mathrm{CO}, \mathrm{NH}_{3}, \mathrm{NO}$, and $\mathrm{NO}_{2}$ donate $0.01(-0.01), 0.09(0.03),-0.00(-0.05)$, and $-0.04(-0.13)$ electrons to the $\mathrm{MoS}_{2}$ $\left(\mathrm{WS}_{2}\right)$ side of the heterobilayer, respectively. According to Figure 3, the pristine $\mathrm{MoS}_{2} / \mathrm{WS}_{2}$ heterobilayer is a semiconductor with a band gap of $1.09 \mathrm{eV}$ (indirect), in agreement with Ref. [31], which is smaller than obtained for both monolayers and both bilayers. The band 


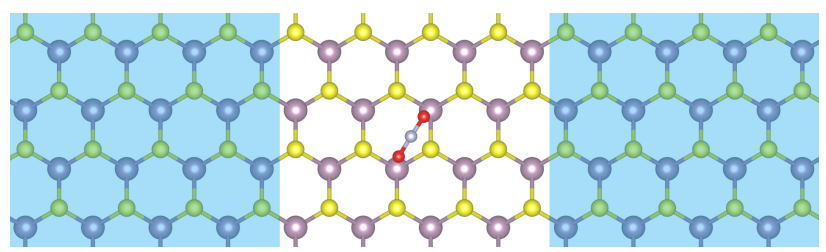

Left Lead Scattering Region Right Lead

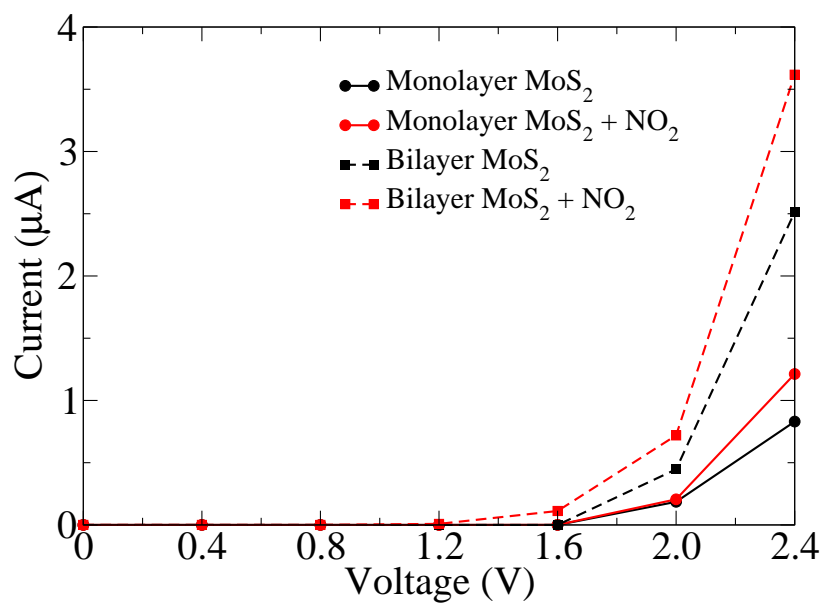

FIG. 4. Transport setup and current-voltage characteristics obtained for monolayer and bilayer $\mathrm{MoS}_{2}$ without and with adsorbed $\mathrm{NO}_{2}$ molecule.

structures given in Figure 3 for adsorption of the different molecules on the $\mathrm{MoS}_{2}$ and $\mathrm{WS}_{2}$ sides of the heterobilayer again indicate that the valence and conduction bands are not significantly affected by the adsorption of non-magnetic $\mathrm{CO}$ and $\mathrm{NH}_{3}$, while the adsorption of paramagnetic $\mathrm{NO}$ and $\mathrm{NO}_{2}$ introduces two and one flat impurity bands inside the band gap, respectively. The behavior thus is the same as in the cases of monolayer and bilayer $\mathrm{MoS}_{2}$.

The adsorption energies of Figure 2 follow the orders $\mathrm{NO}_{2}>\mathrm{NH}_{3}>\mathrm{NO}>\mathrm{CO}$ and bilayer $>$ heterobilayer $>$ monolayer. A comparison of the obtained values to previous results for various two-dimensional materials is given in Table I. Significant differences of our findings to the results of Refs. [24] and [25] for monolayer $\mathrm{MoS}_{2}$ and $\mathrm{WS}_{2}$ show the importance of considering the van der Waals interaction. The fact that bilayers and heterobilayers provide higher adsorption energies than monolayers is a consequence of the interlayer interaction. The binding energy (total energy of the bilayer/heterobilayer minus total energies of the two component layers) per unit cell turns out to be $150 \mathrm{meV}$ for bilayer $\mathrm{MoS}_{2}, 260 \mathrm{meV}$ for bilayer $\mathrm{WS}_{2}$, and $200 \mathrm{meV}$ for the $\mathrm{MoS}_{2} / \mathrm{WS}_{2}$ heterobilayer. We observe that it correlates with the adsorption energy for all gas molecules. For example, $\mathrm{NO}_{2}$ is adsorbed stronger on 
bilayer $\mathrm{WS}_{2}(217 \mathrm{meV})$ than on the $\mathrm{MoS}_{2} / \mathrm{WS}_{2}$ heterobilayer $(213 \mathrm{meV})$ than on monolayer $\mathrm{WS}_{2}(206 \mathrm{meV})$. Therefore, the bilayers and heterobilayers are more effective as gas sensing materials than the monolayers. This observation agrees with the fact that a field effect transistor device based on mechanically exfoliated $\mathrm{MoS}_{2}$ provides a stronger response to NO gas in the multilayer than in the monolayer case [12]. To confirm our conclusions we study the transport properties of monolayer and bilayer $\mathrm{MoS}_{2}$ without and with adsorbed $\mathrm{NO}_{2}$ molecule using the Smeagol code $[37,38]$. The current-voltage characteristics in Figure 4 shows higher current for the bilayer than the monolayer, in agreement with the trends observed for the adsorption energy.

\section{CONCLUSION}

We have studied the adsorption of $\mathrm{CO}, \mathrm{NH}_{3}, \mathrm{NO}$, and $\mathrm{NO}_{2}$ molecules on monolayer, bilayer, and heterobilayer $\mathrm{MoS}_{2}$ and $\mathrm{WS}_{2}$. The calculated adsorption energies show that in each case the gas molecule is physisorbed, with $\mathrm{NO}_{2}$ binding stronger to the sensor materials than the other molecules (which is reflected by enhanced charge transfer). It turns out that the adsorption strength is higher on monolayer and bilayer $\mathrm{WS}_{2}$ than on monolayer and bilayer $\mathrm{MoS}_{2}$, respectively, while the charge transfers are similar. On both sides of the $\mathrm{MoS}_{2} / \mathrm{WS}_{2}$ heterobilayer it is intermediate between bilayer $\mathrm{MoS}_{2}$ and bilayer $\mathrm{WS}_{2}$. Adsorption of non-magnetic $\mathrm{CO}$ and $\mathrm{NH}_{3}$ has little impact on the band structure, whereas the paramagnetic molecules $\mathrm{NO}$ and $\mathrm{NO}_{2}$ induce flat in-gap bands. Bilayer and heterobilayer $\mathrm{MoS}_{2}$ and $\mathrm{WS}_{2}$ not only inherit excellent gas sensing properties from the monolayers but even improve the performance. The same is expected to apply to few layer films (which share the indirect band gap nature).

\section{ACKNOWLEDGEMENT}

We thank Sitansh Sharma for fruitful discussions. The research reported in this publication was supported by funding from King Abdullah University of Science and Technology (KAUST). For computer time, this research used the resources of the Supercomputing Laboratory at KAUST. 


\section{REFERENCES}

[1] Huang J. and Wan Q., Gas Sensors Based on Semiconducting Metal Oxide One-Dimensional Nanostructures, Sensors 9, 9903-9924 (2009).

[2] Fine G. F., Cavanagh L. M., Afonja A., and Binions R., Metal Oxide Semi-Conductor Gas Sensors in Environmental Monitoring, Sensors 10, 5469-5502 (2010).

[3] Pearton S. J., Ren F., Wang Y. L., Chu B. H., Chen K. H., Chang C. Y., Lim W., Lin J. S., and Norton D. P., Recent Advances in Wide Bandgap Semiconductor Biological and Gas Sensors, Prog. Mater. Sci. 55, 1-59 (2010).

[4] Sun Y. F., Liu S. B., Meng F. L., Liu J. Y., Jin Z., Kong L. T., and Liu J. H., Metal Oxide Nanostructures and Their Gas Sensing Properties: A Review, Sensors 12, 2610-2631 (2012).

[5] Su S., Wu W. H., Gao J. M., Lu J. X., and Fan C. H., Nanomaterials-Based Sensors for Applications in Environmental Monitoring, J. Mater. Chem. 22, 18101-18110 (2012).

[6] Korotcenkov G., Metal Oxides for Solid-State Gas Sensors: What Determines Our Choice, Mater. Sci. Eng. B. 139, 1-23 (2007).

[7] Yang W., Gan L., Li H., and Zhai T., Two-Dimensional Layered Nanomaterials for GasSensing Applications, Inorg. Chem. Front. 3, 433-451 (2016).

[8] Kong J., Franklin N. R., Zhou C. W., Chapline M. G., Peng S., Cho K. J., and Dai H. J., Nanotube Molecular Wires as Chemical Sensors, Science 287, 622-625 (2000).

[9] Tricoli A., Righettoni M., and Teleki A., Semiconductor Gas Sensors: Dry Synthesis and Application, Angew. Chem. 49, $7632-7659$ (2010).

[10] Lee K., Gatensby R., McEvoy N., Hallam T., and Duesberg G. S., High-Performance Sensors Based on Molybdenum Disulfide Thin Films, Adv. Mater. 25, 6699-6702 (2013).

[11] Zhou X., Lee S., Xu Z. C., and Yoon J., Recent Progress on the Development of Chemosensors for Gases, Chem. Rev. 115, 7944-8000 (2015).

[12] Li H., Yin Z. Y., He Q. Y., Li H., Huang X., Lu G., Fam D. W. H., Tok A. I. Y., Zhang Q., and Zhang H., Fabrication of Single- and Multilayer $\mathrm{MoS}_{2}$ Film-Based Field-Effect Transistors for Sensing NO at Room Temperature, Small 8, 63-67 (2012).

[13] He Q. Y., Zeng Z. Y., Yin Z. Y., Li H., Wu S. X., Huang X., and Zhang H., Fabrication of Flexible $\mathrm{MoS}_{2}$ Thin-Film Transistor Arrays for Practical Gas-Sensing Applications, Small 8, 2994-2999 (2012). 
[14] Late D. J., Huang Y. K., Liu B., Acharya J., Shirodkar S. N., Luo J. J., Yan A. M., Charles D., Waghmare U. V., Dravid V. P., and Rao C. N. R., Sensing Behavior of Atomically ThinLayered $\mathrm{MoS}_{2}$ Transistors, ACS Nano 7, 4879-4891 (2013).

[15] Yuan W. J., Liu A. R., Huang L., Li C., and Shi G. Q., High-Performance $\mathrm{NO}_{2}$ Sensors Based on Chemically Modified Graphene, Adv. Mater. 25, 766-771 (2013).

[16] Liu B. L., Chen L., Liu G., Abbas A. N., Fathi M., and Zhou C. W., High-Performance Chemical Sensing Using Schottky-Contacted Chemical Vapor Deposition Grown monolayer $\mathrm{MoS}_{2}$ Transistors, ACS Nano 8, 5304-5314 (2014).

[17] Late D. J., Doneux T., and Bougouma M., Single-Layer MoSe 2 Based $\mathrm{NH}_{3}$ Gas Sensor, Appl. Phys. Lett. 105, 233103 (2014).

[18] Schedin F., Geim A. K., Morozov S. V., Hill E. W., Blake P., Katsnelson M. I., and Novoselov K. S., Detection of Individual Gas Molecules Adsorbed on Graphene, Nat. Mater. 6, 652-655 (2007).

[19] Cho B., Hahm M. G., Choi M., Yoon J., Kim A. R., Lee Y. J., Park S. G., Kwon J. D., Kim C. S., Song M., Jeong Y., Nam K. S., Lee S., Yoo T. J., Kang C. G., Lee B. H., Ko H. C., Ajayan P. M., and Kim D. H., Charge-Transfer-Based Gas Sensing Using Atomic-Layer MoS 2 , Sci. Rep. 5, 8052 (2015).

[20] Perkins F. K., Friedman A. L., Cobas E., Campbell P. M., Jernigan G. G., and Jonker B. T., Chemical Vapor Sensing with monolayer $\mathrm{MoS}_{2}$, Nano Lett. 13, 668-673 (2013).

[21] Choi W., Choudhary N., Han G. H., Park J., Akinwande D., and Lee Y. H., Recent Development of Two-Dimensional Transition Metal Dichalcogenides and Their Applications, Materials Today 20, 116-130 (2017).

[22] Mak K. F., Lee C., Hone J., Shan J., and Heinz T. F., Atomically Thin MoS 2 : A New Direct-Gap Semiconductor, Phys. Rev. Lett. 105, 136805 (2010).

[23] Splendiani A., Sun L., Zhang Y., Li T., Kim J., Chim C. Y., Galli G., and Wang F., Emerging Photoluminescence in Monolayer $\mathrm{MoS}_{2}$, Nano Lett. 10, 1271-1275 (2010).

[24] Yue Q., Shao Z., Chang S., and Li J., Adsorption of Gas Molecules on monolayer MoS 2 and Effect of Applied Electric Field, Nanoscale Res. Lett. 8, 425 (2013).

[25] Zhou C., Yang W., and Zhu H., Mechanism of Charge Transfer and Its Impacts on Fermi-Level Pinning for Gas Molecules Adsorbed on monolayer $\mathrm{WS}_{2}$, J. Chem. Phys. 142, 214704 (2015). 
[26] Sun J., Lin N., Ren H., Tang C., Yang L., and Zhao X., Gas Adsorption on $\mathrm{MoS}_{2} / \mathrm{WS}_{2}$ InPlane Heterojunctions and the I-V Response: A First Principles Study, RSC Adv. 6, 1749417503 (2016).

[27] Kresse G. and Joubert D., From Ultrasoft Pseudopotentials to the Projector Augmented-Wave Method, Phys. Rev. B 59, 1758-1775 (1999).

[28] Grimme S., Semiempirical GGA-Type Density Functional Constructed with a Long-Range Dispersion Correction, J. Comput. Chem. 27, 1787-1799 (2006).

[29] Kośmider K. and Fernández-Rossier J., Electronic Properties of the $\mathrm{MoS}_{2}-\mathrm{WS}_{2}$ Heterojunction, Phys. Rev. B 87, 075451 (2013).

[30] He J., Hummer K., and Franchini C., Stacking Effects on the Electronic and Optical Properties of bilayer Transition Metal Dichalcogenides $\mathrm{MoS}_{2}, \mathrm{MoSe}_{2}, \mathrm{WS}_{2}$, and $\mathrm{WSe}_{2}$, Phys. Rev. B 89, 075409 (2014).

[31] Lu N., Guo H., Li L., Dai J., Wang L., Mei W.-N., Wu X., and Zeng X. C., MoS $2 / \mathrm{MX}_{2}$ Heterobilayers: Bandgap Engineering Via Tensile Strain or External Electrical Field, Nanoscale 6, 2879-2886 (2014).

[32] Henkelman G., Arnaldsson A., and Jonsson H., A Fast and Robust Algorithm for Bader Decomposition of Charge Density, Comput. Mater. Sci. 36, 354-360 (2006).

[33] Zhao S., Xue J., and Kang W., Gas Adsorption on $\mathrm{MoS}_{2}$ monolayer From First-Principles Calculations, Chem. Phys. Lett 595, 35-42 (2014).

[34] Amin B., Singh N., and Schwingenschlögl U., Heterostructures of Transition Metal Dichalcogenides, Phys. Rev. B 92, 075439 (2015).

[35] Leenaerts O., Partoens B., and Peeters F. M., Adsorption of $\mathrm{H}_{2} \mathrm{O}, \mathrm{NH}_{3}, \mathrm{CO}, \mathrm{NO}_{2}$, and $\mathrm{NO}$ on Graphene: A First-Principles Study, Phys. Rev. B 77, 125416 (2008).

[36] Pashangpour M., Electronic Structure Calculation of Adsorbate Gas Molecules on a BN Nanolayer, a First Principle Study, Int. Proc. Chemical, Biological and Environmental Engineering 2, 113-116 (2011).

[37] Rocha A. R., García-Suárez V. M., Bailey S. W., Lambert C. J., Ferrer J., and Sanvito S., Towards Molecular Spintonrics, Nat. Mater. 4, 335-339 (2005).

[38] Rungger I. and Sanvito S., Algorithm for the Construction of Self-Energies for Electronic Transport Calculations Based on Singularity Elimination and Singular Value Decomposition, Phys. Rev. B 78, 035407 (2008). 


\section{TOC}

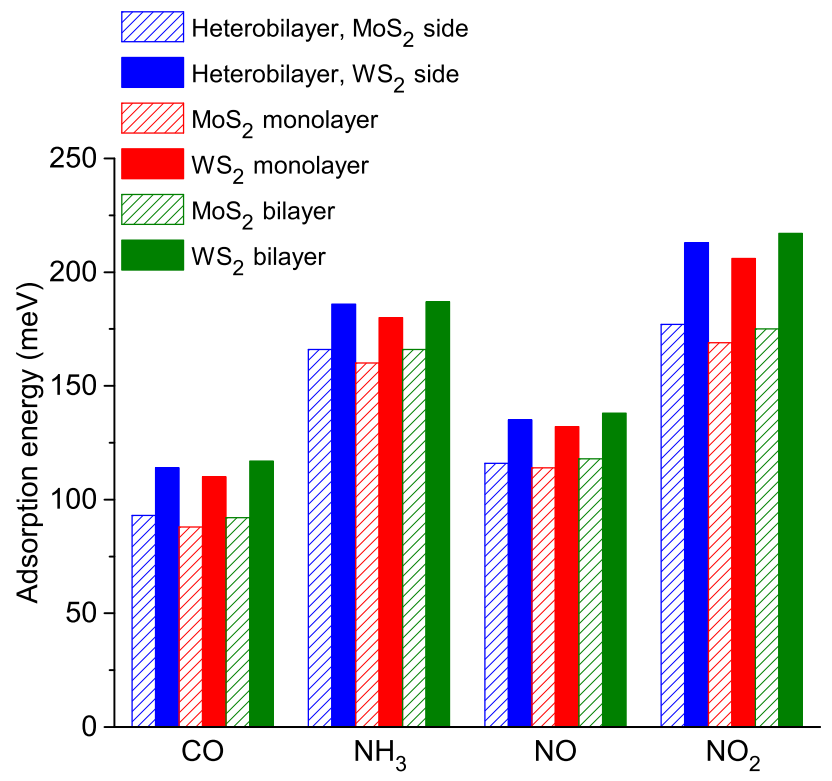

